INT. J. CONTROL, 1988 , vOL. 48 , NO. 5, 2055-2063

\title{
Robust stability of discrete systems
}

\author{
M. E. SEZER † and D. D. ŚILJAK†
}

The objective of this paper is to show how to choose a Liapunov function to obtain the best and sometimes exact estimates of the degree of exponential stability for linear time-invariant discrete systems. The choice is interesting because it is also shown that it provides the largesı robustness bounds on non-linear lime-varying perturbations which can be established by either norm-like or quadratic Liapunov functions. By applying the results obtained to large-scale systems, where the role of perturbations is played by the interconnections among the subsystems, the least conservative stability conditions are derived for the overall system which are available in the context of vector Liapunov functions and $M$-matrices.

\section{Introduction}

An attractive feature of the Liapunov direct method is the fact that when we cstablish stability of a given dynamic system by a Liapunov function we can use the function to estimate the rate of decay of the iransient response of the system. In linear timc-invariant systems this amounts to providing an estimate of the degree of (exponential) stability which, in the case of continuous-time systems, is synonymous with an estimate of the real part of the maximal eigenvalue of the system matrix. An added significance of the degree of stability is that it yields directly the robustness bounds on the non-linear time-varying perturbations which the system can tolerate without going unstable (Kalman and Bertram 1960).

In large-scale dynamic systems, the interconnection terms can be regarded as perturbations of the subsystem dynamics. Then, the degree of stability of each subsystem becomes a measure of how high the interconnection level may be for a vector-type of Liapunov function to guarantee stability of the overall system. For this reason, an optimal choice of the norm-like Liapunov function was proposed (Siljak 1976) which provides the exact cstimate of the degree of stability of a subsystem when it is represented by a continuous-time linear time-invariant model. This estimate, however, requires the system matrix to be transformable into a semisimple form. If this is not the case, one may prefer to stay in the original coordinate frame and choose the Liapunov function proposed by Patel and Toda (1980) at the expense of making the best, but not exact, estimate of the degree of exponential stability of the system.

The objective of this paper is to reproduce the above described developinent in the context of discrete-time systems. There are some expected similarities between the two classes of systems, but there are a few notable distinctions. While the way in which one solves the Liapunov matrix equation to get the best function is the same, the normlike Liapunov functions are generally better than the quadratic forms when discretetime systems are considered. If the system matrix is in a semi-simple form, the two typcs of functions produce the exact estimate of the spectral radius of the matrix,

Received 25 January 1988.

+ Department of Electrical and Electronics Engineering. Bilkent University, Ankara, Turkey.

$\ddagger$ B\&M Swig Professor, Santa Clara University, Santa Clara, CA 95053, U.S.A. 
which is similar to the result available for continuous-time systems. The most pleasing similarity, which we were able to establish in this context, is the fact that by maximizing the estimate of the degree of stability we maximize at the same time the robustness bounds on the additive perturbations which may act on the system. This fact is of particular importance in maximizing chances to establish exponential stability of large-scale discrete-time systems using the methods of vector Liapunov functions and a discretc version of the comparison principle formulated by Grujić and Siljak (1973, 1974). The results can be used as a basis for robust stabilization of large-scale discrete systems by decentralizcd feedback in much the same way it was done in the continuous case (Siljak 1978). This is a welcome development because of the recent widespread orientation in practical applications toward distributed controllers with parallel computational capabilities.

\section{Degree of stability}

Consider a discrete-time linear time-invariant system

$$
\text { S: } x(k+1)=A x(k)
$$

where $x(k) \in \mathbf{R}^{n}$ is the state of $\mathbf{S}$ at time $k \in \mathbf{T}_{+}=\{0,1, \ldots\}$, and $A$ is a constant $n \times n$ matrix. We denote the solution at time $k$ of $(2.1)$ starting from $x_{0}=x(0)$ by $x\left(k ; x_{0}\right)$ or, simply, by $x(k)$. We state the following.

\section{Definition 2.2}

The system $\mathbf{S}$ is said to be exponentially stable if there exist numbers $\Pi>0$ and $0 \leqslant \rho<1$ such that

$$
\|x(k)\| \leqslant \Pi\left\|x_{0}\right\| \rho^{k} \quad \forall k \in \mathbf{T}_{+}, \forall x_{0} \in \mathbf{R}^{n}
$$

In the following development, we use the cuclidean norm $\|x\|=\left(x^{\top} x\right)^{1 / 2}$ and the induced matrix norm $\|\cdot\|=\sigma_{M}(\cdot)$, where $\sigma_{M}(\cdot)$ is the maximum singular value of the indicated matrix.

We assume that $\mathbf{S}$ is exponentially stable and consider $\rho$ in (2.3) to be the spectral radius of $A$. This value of $\rho$ we term the degree of stability of $\mathbf{S}$ and aim at computing an estimate $\dot{\rho}$ using the Liapunov direct method. We recall that (see Kalman and Bertram, 1960) $\mathbf{S}$ is asymptotically stable if and only if for any symmetric positive definitc $G$ there cxists a unique symmetric positive definite matrix $H$ which satisfies the Liapunov equation

$$
A^{\top} H A-H=-G
$$

The solution of (2.4) is

$$
H=\sum_{i=0}^{\infty}\left(A^{\top}\right)^{i} G A^{i}
$$

To compute $\hat{\rho}$ we consider two types of Liapunov functions based on (2.4), namely, quadratic and norm-like functions. We start with the quadratic form

$$
V(x)=x^{\top} H x
$$

which satisfies the inequalities

$$
\sigma_{m}(H)\|x\|^{2} \leqslant V(x) \leqslant \sigma_{M}(H)\|x\|^{2}
$$


where $\sigma_{m}(\cdot)$ is the minimum singular value of the indicated matrix. We compute $\Delta V[x(k)]_{\mathrm{S}}=V[x(k+1)]-V[x(k)]$ with respect to (2.1) and get the inequality

$$
\Delta v[x(k)]_{s} \leqslant-\sigma_{m}(G)\|x(k)\|^{2} \quad \forall x \in \mathbf{R}^{n}
$$

From (2.7) and (2.8), we get

$$
V[x(k+1)] \leqslant\left[1-\frac{\sigma_{m}(G)}{\sigma_{M}(H)}\right] V[x(k)]
$$

so that

$$
V[x(k+1)] \leqslant\left[1-\frac{\sigma_{m}(G)}{\sigma_{M}(H)}\right]^{k} V\left(x_{0}\right)
$$

Using (2.7) and (2.10) we obtain

$$
\|x(k)\| \leqslant \frac{\sigma_{M}^{1 / 2}(H)}{\sigma_{m}^{1 / 2}(H)}\left(\left[1-\frac{\sigma_{m}(G)}{\sigma_{M}(H)}\right]^{1 / 2}\right)^{k}\left\|x_{0}\right\|
$$

Comparing (2.11) with (2.3), we get the estimate

$$
\hat{\rho}_{V}(G)=\left[1-\frac{\sigma_{m}(G)}{\sigma_{M}(H)}\right]^{1 / 2}
$$

Our interest is to solve the following.

Problem 2.13

$$
\min _{G} \hat{\rho}_{V}(G)
$$

subject to

$$
A^{\top} H A-H=-G
$$

\section{Solution}

From (2.5), we write

$$
x^{\top} H x \geqslant \sigma_{m}(G) x^{\top}\left[\sum_{i=0}^{\infty}\left(A^{\top}\right)^{i} A^{i}\right] x=\sigma_{m}(G) x^{\top} H^{*} x
$$

where $H^{*}$ is the solution of (2.3) corresponding to $G=I$, and get

$$
\sigma_{M}(H) x^{\mathrm{T}} x \geqslant \sigma_{m}(G) x^{\mathrm{T}} H^{*} x \quad \forall x \in \mathbf{R}^{n}
$$

to arrive at

$$
\frac{\sigma_{m}(G)}{\sigma_{M}(H)} \leqslant \frac{x^{\top} x}{x^{\top} H^{*} x} \quad \forall x \in \mathbf{R}^{n}-\{0\}
$$

Inequality (2.16) implies

$$
\hat{\rho}_{V}(G) \geqslant\left[1-\frac{1}{\sigma_{M}\left(H^{*}\right)}\right]^{1 / 2}=\hat{\rho}_{V}(I)
$$

Therefore, the unique minimum $\hat{\rho}_{v}^{*}=\hat{\rho}_{V}(I)$ is achieved at $G^{*}=I$. 
We turn our attention to a norm-like Liapunov function

$$
v(x)=\left(x^{\top} H x\right)^{1 / 2}
$$

which satisfies the inequalities

$$
\sigma_{m}^{1 / 2}(H)\|x\| \leqslant v(x) \leqslant \sigma_{M}^{1 / 2}(H)\|x\|
$$

and compute

$$
\begin{aligned}
\Delta v[x(k)]_{\mathrm{s}} & =\left[x^{\mathrm{\top}}(k+1) H x(k+1)\right]^{1 / 2}-\left[\mathrm{x}^{\mathrm{T}}(k) H x(k)\right]^{1 / 2} \\
& =\frac{x^{\mathrm{T}}(k+1) H x(k+1)-x^{\mathrm{T}}(k) H x(k)}{\left[x^{\mathrm{T}}(k+1) H x(k+1)\right]^{1 / 2}+\left[x^{\mathrm{T}}(k) H x(k)\right]^{1 / 2}} \\
& \leqslant-\frac{x^{\mathrm{T}}(k) G x(k)}{\left[x^{\mathrm{T}}(k)(H-G) x(k)\right]^{1 / 2}+\left[x^{\mathrm{T}}(k) H x(k)\right]^{1 / 2}} \\
& \leqslant-\frac{\sigma_{m}(G)}{\sigma_{M}^{1 / 2}(H-G)+\sigma_{M}^{1 / 2}(H)}\|x(k)\| \quad \forall x \in \mathbf{R}^{n}
\end{aligned}
$$

By following the steps in arriving at (2.11), we get the estimate

$$
\hat{\rho}_{\nu}(G)=1-\frac{\sigma_{m}(G)}{\sigma_{M}(H)+\sigma_{M}^{1 / 2}(H) \sigma_{M}^{1 / 2}(H-G)}
$$

Now, we solve the following.

Problem 2.22

$$
\min _{G} \hat{\rho}_{v}(G)
$$

subject to

$$
A^{\top} H A-H=-G
$$

\section{Solution}

From (2.5), we write

$$
x^{\mathrm{T}}(H-G) x \geqslant \sigma_{m}(G) x^{\mathrm{T}}\left[\sum_{i=1}^{\infty}\left(A^{\mathrm{T}}\right)^{i} A^{\mathrm{i}}\right] x=\sigma_{m}(G) x^{\mathrm{T}}\left(H^{*}-I\right) x
$$

Using (2.14) together with (2.23), and proceeding as in the solution of Problem 2.13, we get

$$
\begin{aligned}
& \frac{\sigma_{m}(G)}{\sigma_{M}(H)+\frac{\sigma_{M}^{1 / 2}(H) \sigma_{M}^{1 / 2}(H-G)}{}} \\
& \quad \leqslant \frac{x^{\mathrm{T}} x}{x^{\top} H^{*} x+\left(x^{\mathrm{T}} H^{*} x\right)^{1 / 2}\left[x^{\mathrm{T}}\left(H^{*}-l\right) x\right]^{1 / 2}} \quad \forall x \in \mathbf{R}^{\mathrm{n}}-\{0\}
\end{aligned}
$$

Noting that all three terms in the denominator at the right side of (2.24) achieve their maximum at the same point, we get

$$
\hat{\rho}_{v}(G) \geqslant 1-\frac{1}{\sigma_{M}\left(H^{*}\right)+\sigma_{M}^{1 / 2}\left(H^{*}\right) \sigma_{M}^{1 / 2}\left(H^{*}-l\right)}=\hat{\rho}_{v}(I)
$$


Therefore, the unique minimum $\hat{\rho}_{v}^{*}=\bar{\rho}_{v}(I)$ is achieved as in Problem 2.13 at $G^{*}=I$.

The two estimates $\hat{\rho}_{v}(G)$ and $\hat{\rho}_{v}(G)$ compare as follows.

Proposition 2.26

$\hat{\rho}_{v}(G) \leqslant \hat{\rho}_{V}(G)$ for all $G$ with equality holding for $G=I$.

Proof

From the obvious inequality $\sigma_{M}(H-G) \leqslant \sigma_{M}(H)-\sigma_{m}(G)$, we obtain

$$
\begin{aligned}
\sigma_{M}(H)-\left[\sigma_{M}^{2}(H)-\sigma_{M}(H) \sigma_{m}(G)\right]^{1 / 2} & \\
& \leqslant \Psi \leqslant \sigma_{M}(H)+\left[\sigma_{M}^{2}(H)-\sigma_{M}(H) \sigma_{M}(G)\right]^{1 / 2}
\end{aligned}
$$

where $\Psi=\sigma_{M}(H)+\sigma_{M}^{1 / 2}(H) \sigma_{M}^{1 / 2}(H-G)$. Using (2.27), we obtain the inequality

$$
\Psi^{2}-2 \sigma_{M}(H) \Psi+\sigma_{M}(H) \sigma_{M}(G) \leqslant 0
$$

with equality holding for $G=I$. Finally, from (2.28) we get

$$
\hat{\rho}_{v}(G)=\left(1-2 \frac{\sigma_{m}(G)}{\Psi}+\frac{\sigma_{m}^{2}(G)}{\Psi^{2}}\right)^{1 / 2} \leqslant\left(1-\frac{\sigma_{m}(G)}{\sigma_{M}(H)}\right)^{1 / 2}=\hat{\rho}_{v}(G)
$$

with equality for $G=I$.

It is interesting to note that, unlike continuous-time systems (Šijak 1978), the two Liapunov functions do not produce the same estimate of the degree of stability of $\mathbf{S}$, and the norm-like function is superior to the corresponding quadratic form.

To get a feeling of how conservative the obtained estimates are we consider the special form of $A$ for which it is known (Siljak 1978) that in the continuous-time case the Liapunov method provides the exact estimate of the real part of its largest eigenvalue. Suppose $A$ is semisimple having the form

$$
A=\operatorname{diag}\left\{\Lambda_{1}, \Lambda_{2}, \ldots, \Lambda_{p}, \mu_{2 p+1}, \ldots, \mu_{n}\right\}
$$

where

$$
\Lambda_{i}=\left[\begin{array}{cc}
\left|\mu_{i}\right| \cos \theta_{i} & -\left|\mu_{i}\right| \sin \theta_{i} \\
\left|\mu_{i}\right| \sin \theta_{i} & \left|\mu_{i}\right| \cos \theta_{i}
\end{array}\right], \quad i=1,2, \ldots, p
$$

Choosing $G=I$, we get from (2.4)

$$
H^{*}=\operatorname{diag}\left\{H_{1}, H_{2}, \ldots, H_{p}, h_{2 p+1}, \ldots . h_{n}\right\}
$$

where

$$
H_{i}=\frac{1}{1-\mu_{i}^{2}} l_{2}, \quad h_{i}=\frac{1}{1-\mu_{i}^{2}}
$$

We observe that $\hat{\rho}_{v}^{*}=\hat{\rho}_{v}^{*}=\max \left|\mu_{i}\right|=\rho$, and conclude that both types of functions produce the exact estimate of the spectral radius of $A$, which is the maximal degree of stability of the corresponding discrete-time system as it was the case with continuoustime systems. 


\section{Robustness bounds}

We consider a perturbed system

$$
\mathbf{S}: x(k+1)=A x(k)+h[k, x(k)]
$$

where the nonlinear perturbation $h: \mathbf{T}_{+} \times \mathbf{R}^{n} \rightarrow \mathbf{R}^{n}$ is bounded as

$$
\|h(k, x)\| \leqslant \xi\|x\| \quad \forall k \in \mathbf{T}_{+}, \forall x \in \mathbf{R}^{n}
$$

and $\xi \geqslant 0$. We assume that the nominal system $\mathbf{S}$ of $(2.1)$ is exponentially stable with degree $\rho$, and aim at computing the largest number $\hat{\xi}$ for which the perturbed system $\mathbf{S}$ is also exponentially stable. We use both types of Liapunov functions and relate the robustness bound $\hat{\xi}$ to the estimate $\hat{\rho}$ of the degree of stability of $\mathbf{S}$.

For the function $V(x)$ of $(2.6)$, we compute

$$
\begin{aligned}
\Delta V(x)_{\mathrm{s}}= & -x^{\mathrm{T}} G x+2 x^{\mathrm{T}} A^{\mathrm{T}} H h+h^{\mathrm{T}} H h \\
\leqslant & -x^{\mathrm{T}} G x+2\left(x^{\mathrm{T}} A^{\mathrm{T}} H A x\right)^{1 / 2}\left(h^{\mathrm{T}} H h\right)^{1 / 2}+h^{\mathrm{T}} H h \\
\leqslant & -\left[\sigma_{m}(G)-2 \sigma_{M}^{1 / 2}(H) \sigma_{M}^{1 / 2}(H-G) \xi-\sigma_{M}(H) \xi^{2}\right]\|x\|^{2} \\
& \forall k \in \mathbf{T}_{+}, \forall x \in \mathbf{R}^{\mathrm{n}}
\end{aligned}
$$

where the inequality $p^{\top} H q \leqslant\left(p^{\top} H p\right)^{1 / 2}\left(q^{\top} H q\right)^{1 / 2}$ was used for $p=A x$ and $q=h$, together with (3.2). From (3.3), we observe that $\Delta V(x)<0$ when $\xi<\xi_{V}(G)$ where

$$
\xi_{V}(G)=\frac{\left[\sigma_{m}(G)+\sigma_{M}(H-G)\right]^{1 / 2}-\sigma_{M}^{1 / 2}(H-G)}{\sigma_{M}^{1 / 2}(H)}
$$

Note that the robustness bound corresponding to $G=I$ is

$$
\hat{\xi}_{V}^{*}=1-\hat{\rho}_{V}^{*}
$$

When we consider the norm-like function $v(x)$ of $(2.18)$, we compute

$$
\begin{aligned}
\Delta v(x)_{\mathrm{s}} & =v(A x+h)-v(x) \\
& \leqslant v(A x)-v(x)+|v(A x+h)-v(A x)| \\
& \leqslant \Delta v(x)_{\mathrm{S}}+|v(A x+h)-v(A x)| \\
& \leqslant-\left[\frac{\sigma_{m}(G)}{\sigma_{M}^{1 / 2}(H-G)+\sigma_{M}^{1 / 2}(H)}-\sigma_{M}^{1 / 2}(H) \xi\right]\|x\| \quad \forall k \in \mathbf{T}_{+}, \forall x \in \mathbf{R}^{n}
\end{aligned}
$$

where the inequality $\left|\left(p^{\mathrm{T}} H p\right)^{1 / 2}-\left(q^{\mathrm{T}} H q\right)^{1 / 2}\right| \leqslant \sigma_{M}^{1 / 2}(H)\|p-q\|$ was used for $p=A x+h$ and $q=A x$, together with (2.20) and (3.2). From (3.6), we obtain the robustness bound

$$
\xi_{p}(G)=\frac{\sigma_{m}(G)}{\sigma_{M}(H)+\sigma_{M}^{1 / 2}(H) \sigma_{M}^{1 / 2}(H-G)}
$$

and note that

$$
\xi_{1,}(G)=1-\hat{\rho}_{v}(G)
$$

can be regarded as the stability margin of $\mathbf{S}$.

We remark that the maximization of the robustness bound is reduced to the minimization of the estimate of the stability degree, which was solved in the preceding section. We recall, however, that the best (exact) estimate is achieved when the matrix 
$A$ is semisimple, which motivates a similarity transformation of $A$ to a semisimple form. The transformation, however, effects the perturbation function $h$, and the improvement of the robustness bound depends ultimately upon the trade-off between the gain in the estimate of the stability margin and the change of size of $h$.

We should also note that there are other ways of producing Liapunov functions for robustness analysis of discrete systems (see, e.g., Kalman and Bertram 1960, Geromel and Da Cruz 1987), but they may produce robustness bounds which are far from being the best available in this context.

\section{Interconnected systems}

We finally consider the perturbed system $\mathbf{S}$ of $(3.1)$ as an interconnected system

$$
\text { S: } \quad x_{i}(k+1)=A_{i} x_{i}(k)+h_{i}[k, x(k)], \quad i \in \mathbf{N}
$$

which is composed of $N$ subsystems

$$
\mathbf{S}_{i}: \quad x_{i}(k+1)=\boldsymbol{A}_{i} x_{i}(k), \quad i \in \mathbf{N}
$$

where $x_{i}(k) \in \mathbf{R}^{n_{1}}$ is the state of $\mathbf{S}_{i}$ at $k \in \mathbf{T}_{+}, x(k)=\left[x_{1}^{\mathrm{T}}(k), x_{2}^{\mathrm{T}}(k), \ldots, x_{N}^{\mathrm{T}}(k)\right]^{\top}$ is the state of the overall system $\mathbf{S}, A_{i}$ is an $n_{i} \times n_{i}$ constant matrix, $h_{i}: \mathbf{T}+\times \mathbf{R}^{n} \rightarrow \mathbf{R}^{n_{1}}$ is the ith interconnection function, and $\mathbf{N}=\{1,2, \ldots, N\}$.

We assume that each $\mathbf{S}_{i}$ is exponentially stable, that is, for any symmetric positive definite $G_{i}$, there exists a unique symmetric positive definite solution $H_{i}$ to the Liapunov equation

$$
A_{i}^{\mathrm{T}} H_{i} A_{i}-H_{i}=-G_{i}, \quad i \in \mathbf{N}
$$

We also assume that the interconnections $h_{i}(k, x)$ satisfy the inequalities

$$
\left\|h_{i}(k, x)\right\| \leqslant \sum_{j \in \mathbf{N}} \xi_{i j}\left\|x_{j}\right\| \quad \forall k \in \mathbf{T}_{+}, \forall x \in \mathbf{R}^{n}, \forall i \in \mathbf{N}
$$

for some non-negative numbers $\xi_{i j}$.

To establish exponential stability of the system $\mathbf{S}$ from the same property of the subsystems $\mathbf{S}_{i}$, we start first with subsystem Liapunov functions of the quadratic type,

$$
V_{i}\left(x_{i}\right)=x_{i}^{\top} H_{i} x_{i}
$$

and compute $\Delta V_{i}\left(x_{i}\right)_{\mathrm{s}}$ imitating (3.3),

$$
\begin{aligned}
\Delta V_{i}\left(x_{i}\right)_{\mathrm{s}} \leqslant & -\left[\sigma_{m}\left(G_{i}\right)\left\|x_{i}\right\|^{2}-2 \sigma_{M}^{1 / 2}\left(H_{i}\right) \sigma_{M}^{1 / 2}\left(H_{i}-G_{i}\right)\left\|h_{i}\right\|\left\|x_{i}\right\|-\sigma_{M}\left(H_{i}\right)\left\|h_{i}\right\|^{2}\right] \\
\leqslant & -\left[\sigma_{m}\left(G_{i}\right)+\sigma_{M}\left(H_{i}-G_{i}\right)\right]\left\|x_{i}\right\|^{2} \\
& +\left[\sigma_{M}^{1 / 2}\left(H_{i}\right)\left\|h_{i}\right\|+\sigma_{M}^{1 / 2}\left(H_{i}-G_{i}\right)\left\|x_{i}\right\|\right]^{2} \\
\leqslant & -\sigma_{M}\left(H_{i}\right)\left[\alpha_{i}^{2}\left\|x_{i}\right\|^{2}+\left(\beta_{i}\left\|x_{i}\right\|+\sum_{j \in \mathbb{N}} \xi_{i j}\left\|x_{j}\right\|\right)^{2}\right] \\
& \forall k \in \mathbf{T}_{+}, \forall x \in \mathbf{R}^{n}, \forall i \in \mathbf{N}
\end{aligned}
$$

where

$$
\alpha_{i}=\left(\frac{\sigma_{m}\left(G_{i}\right)+\sigma_{M}\left(H_{i}-G_{i}\right)}{\sigma_{M}\left(H_{i}\right)}\right)^{1 / 2}, \quad \beta_{i}=\left(\frac{\sigma_{M}\left(H_{i}-G_{i}\right)}{\sigma_{M}\left(H_{i}\right)}\right)^{1 / 2}
$$

We choose the function

$$
v_{V}(x)=\sum_{i \in \mathbf{N}} d_{i} V_{i}\left(x_{i}\right)
$$


as a candidate for Liapunov function for $\mathbf{S}$, and compute $\Delta v_{V}(x)_{S}$ using (4.6),

$$
\Delta v_{v}(x)_{\mathrm{s}} \leqslant-w^{\mathrm{T}}(x) \tilde{W}_{v} w(x), \quad \forall x \in \mathbf{R}^{n}
$$

where $w(x)=\left(\left\|x_{1}\right\|,\left\|x_{2}\right\|, \ldots,\left\|x_{N}\right\|\right)^{\mathrm{T}}$ and

$$
\tilde{W}_{v}=C \tilde{D} C-B^{T} \tilde{D} B
$$

with $C=\operatorname{diag}\left\{\alpha_{1}, \alpha_{2}, \ldots, \alpha_{N}\right\}, \tilde{D}=\operatorname{diag}\left\{d_{1} \sigma_{M}\left(H_{1}\right), d_{2} \sigma_{M}\left(H_{2}\right), \ldots, d_{N} \sigma_{M}\left(H_{N}\right)\right\}$, and $B=\left(b_{i j}\right)$ where

$$
b_{i j}= \begin{cases}\beta_{i}+\xi_{i i}, & i=j \\ \xi_{i j}, & i \neq j\end{cases}
$$

We now define a matrix

$$
W_{v}=C-B
$$

and prove the following.

\section{Proposition 4.13}

The system $\mathbf{S}$ is exponentially stable if $W_{V}$ is an $M$-matrix.

\section{Proof}

We note that if $W_{V}$ is an $M$-matrix then there exists a diagonal matrix $\tilde{D}$ with positive elements such that $\tilde{W}_{V}$ is positive definite (Tartar 1971). The proof then follows from inequality (4.9).

The development leading to Proposition 4.13 is a straightforward application of the concept of vector Liapunov functions (see Siljak 1978, Araki 1978, Michel 1983, Vidyasagar 1986). An interesting part of the analysis, however, is the quantitative aspect of the test matrix $W_{v}=\left(w_{i j}^{V}\right)$ having the elements

$$
w_{i j}^{v}= \begin{cases}\xi_{V}\left(G_{i}\right)-\xi_{i i}, & i=j \\ -\xi_{i j}, & i \neq j\end{cases}
$$

where $\xi_{v}\left(G_{i}\right)$ is the robustness bound for the subsystem $\mathbf{S}_{i}$ defined in (3.4). To maximize the chance of proving that $W_{V}$ is an $M$-matrix, we maximize the robustness bound by choosing $G_{i}=I_{i}$ to get $\xi_{V}\left(I_{i}\right)=1-\hat{\rho}_{i}^{*}$.

When we consider norm-like Liapunov functions

$$
v_{i}\left(x_{i}\right)=\left(x_{i}^{\mathrm{T}} H_{i} x_{i}\right)^{1 / 2}
$$

for the subsystems, we compute

$$
\begin{aligned}
\Delta v_{i}\left(x_{i}\right)_{s} & \leqslant-\sigma_{M}^{1 / 2}\left(H_{i}\right)\left[\xi_{v}\left(G_{i}\right)\left\|x_{i}\right\|-\left\|h_{i}\right\|\right] \\
& \leqslant-\sigma_{M}^{1 / 2}\left(H_{i}\right)\left[\xi_{v}\left(G_{i}\right)\left\|x_{i}\right\|-\sum_{j \in \mathbf{N}} \xi_{i j}\left\|x_{j}\right\|\right] \quad \forall k \in \mathbf{T}_{+}, \forall x \in \mathbf{R}^{n}, \forall i \in \mathbf{N}
\end{aligned}
$$

where $\xi_{v}\left(G_{i}\right)$ is the stability margin of the subsystem $\mathbf{S}_{i}$ defined in (3.7). We again choose the same type of function as in (4.8),

$$
v_{v}(x)=\sum_{i \in \mathrm{N}} d_{i} v_{i}\left(x_{i}\right)
$$


and compute

$$
\Delta v_{v}(x)_{\mathrm{S}} \leqslant-J^{\mathrm{T}} W_{v} w(x) \quad \forall x \in \mathbf{R}^{n}
$$

where $d=\left[d_{1} \sigma_{M}^{1 / 2}\left(H_{1}\right), d_{2} \sigma_{M}^{1 / 2}\left(H_{2}\right), \ldots, d_{N} \sigma_{M}^{1 / 2}\left(H_{N}\right)\right]^{\mathrm{T}}$, and with $\left(w_{i j}^{v}\right)$ where

$$
w_{i j}^{v}= \begin{cases}\xi_{v}\left(G_{i}\right)-\xi_{i i}, & i=j \\ -\xi_{i j}, & i \neq j\end{cases}
$$

Proposition 4.20

The system $\mathbf{S}$ is exponentially stable if $W_{v}$ is an $M$-matrix.

Proof

If the matrix $W_{v}$ is an $M$-matrix, then there exists a vector $d$ with positive elements such that $J^{\mathrm{T}} W_{v}$ has positive elements (Siljak 1978). The proof follows from (4.18).

As in the case of quadratic Liapunov functions, the best choice for the subsystem Liapunov functions is $G_{i}=I_{i}$, which produces the maximum stability margin for $\mathbf{S}_{i}$. Note that for this choice of $G_{i}, W_{v}=W_{v}$.

\section{Conclusions}

It has been shown that we can choose norm-like and quadratic Liapunov functions that maximize the estimates of the degree of stability for linear discrete systems and, at the same time, produce the best robustness bounds on non-linear time-varying perturbations which are available in this context. Since the interconnections in systems composed of interacting subsystems can be regarded as perturbations of the subsystem dynamics, the obtained results have direct applications to stability analysis of large-scale systems via the concepts of vector Liapunov functions and $M$-matrices.

\section{ACKNOWLEDGMENTS}

This research was supported in part by the National Science Foundation under the Grant ECS-8813273, and in part by the Science and Engineering Center of the Bilkent University, Ankara, Turkey.

\section{REFERENCES}

ARak1, M., 1978, I.E.E.E. Trans. autom. Control, 23, 129.

Geromel, J. C., and DA Cruz, J. J., 1987, I.E.E.E. Trans. autom. Control, 32, 703.

Grujle, L. T., and Siljak, D. D., 1973, I.E.E.E. Trans. autom. Control, 18, 522; 1974, Int. J. Control, 19, 481.

Kalman, R. E., and Bertram, J. E., 1960, Trans. Am. Soc. mech. Engrs, Pt D, J. bas. Engng, 82, 394.

MiChel, A. N., 1983, I.E.E.E. Trans. Circuit Systems, 30, 326.

PATEL, R. V., and TODA, M., 1980, Quantitative measures of robustness for multivariable systems. Proc. Joint Automatic Control Conf., San Francisco, CA, Paper TP8-A.

SILJAK, D. D., 1976, Automatica, 12, 309; 1978, Large-Scale Dynamic Systems: Stability and Structure (New York: North-Holland).

Tartar, L., 1971, Rev. Autom. Inf. Res. Oper., 5, 127.

Vidyasagar, M., 1986, Proc. Inst. elect. electron. Engrs, 74, 1060. 